\title{
Oscillation of second-order damped differential equations
}

\author{
Xiaoling Fu ${ }^{1,2}$, Tongxing $\mathrm{Li}^{1}$ and Chenghui Zhang ${ }^{1 *}$
}

\section{"Correspondence:}

zchui@sdu.edu.cn

'School of Control Science and Engineering, Shandong University, Jinan, Shandong 250061, P.R. China Full list of author information is available at the end of the article

\begin{abstract}
We study oscillatory behavior of a class of second-order differential equations with damping under the assumptions that allow applications to retarded and advanced differential equations. New theorems extend and improve the results in the literature. Illustrative examples are given.
\end{abstract}

MSC: $34 \mathrm{C} 10 ; 34 \mathrm{~K} 11$

Keywords: oscillation; functional differential equation; damping term

\section{Introduction}

This paper is concerned with oscillation of solutions to a second-order differential equation with damping

$$
\left(r(t) x^{\prime}(t)\right)^{\prime}+p(t) x^{\prime}(t)+q(t) f(x(\tau(t)))=0
$$

where $t \geq t_{0}>0, r \in \mathrm{C}^{1}\left(\left[t_{0},+\infty\right),(0,+\infty)\right), p, q, \tau \in \mathrm{C}\left(\left[t_{0},+\infty\right), \mathbb{R}\right), q(t) \geq 0, q$ does not vanish eventually, $f \in \mathrm{C}(\mathbb{R}, \mathbb{R}), f(x) / x \geq \mu$ for some $\mu>0$ and for all $x \neq 0$. Throughout, we assume that solutions of (1.1) exist for any $t \geq t_{0}$. A solution $x$ of (1.1) is termed oscillatory if it has arbitrarily large zeros; otherwise, we call it nonoscillatory. Equation (1.1) is said to be oscillatory if all its solutions are oscillatory.

During the past decades, the questions regarding the study of oscillatory properties of differential equations with damping or distributed deviating arguments have become an important area of research due to the fact that such equations arise in many real life problems; see the research papers [1-26] and the references cited therein. In particular, secondorder damped differential equations are used in the study of NVH of vehicles. In what follows, we present the background details that motivate the contents of this paper. Yan [25] established an important extension of the celebrated Kamenev oscillation criterion [27] for a second-order damped equation

$$
\left(r(t) x^{\prime}(t)\right)^{\prime}+p(t) x^{\prime}(t)+q(t) x(t)=0 .
$$

Rogovchenko [19] and Rogovchenko and Tuncay [20] studied a nonlinear damped equation

$$
\left(r(t) x^{\prime}(t)\right)^{\prime}+p(t) x^{\prime}(t)+q(t) f(x(t))=0 .
$$

○2013 Fu et al.; licensee Springer. This is an Open Access article distributed under the terms of the Creative Commons Attribution License (http://creativecommons.org/licenses/by/2.0), which permits unrestricted use, distribution, and reproduction in any medium, provided the original work is properly cited. 
Rogovchenko and Tuncay [21] extended the results of [20] to a general nonlinear damped equation

$$
\left(r(t) \psi(x(t)) x^{\prime}(t)\right)^{\prime}+p(t) x^{\prime}(t)+q(t) f(x(t))=0
$$

In $[8,15]$, the authors investigated (1.1) under the assumptions that $r, p, q \in \mathrm{C}\left(\left[t_{0},+\infty\right)\right.$, $(0,+\infty)), \tau(t) \leq t$, and $\tau^{\prime}(t)>0$. The natural question now is: Can one extend the results of [20] to functional equation (1.1)? The purpose of this paper is to give an affirmative answer to this question.

\section{Main results}

In the sequel, all functional inequalities are supposed to be satisfied for all sufficiently large $t$. We use the notation

$$
\mathbb{D}:=\left\{(t, s): t_{0} \leq s \leq t<+\infty\right\} \quad \text { and } \quad \mathbb{D}_{0}:=\left\{(t, s): t_{0} \leq s<t<+\infty\right\} .
$$

We say that a continuous function $H: \mathbb{D} \rightarrow[0,+\infty)$ belongs to the class $\mathcal{W}$ if:

(i) $H(t, t)=0$ for $t \geq t_{0}$ and $H(t, s)>0$ for $(t, s) \in \mathbb{D}_{0}$;

(ii) $H$ has a nonpositive continuous partial derivative with respect to the second variable satisfying, for some locally integrable continuous function $h$,

$$
\frac{\partial}{\partial s} H(t, s)=-h(t, s)(H(t, s))^{\frac{1}{2}}
$$

Using ideas exploited by Rogovchenko and Tuncay [20], we study (1.1) in the cases where

$$
\tau(t) \leq t
$$

and

$$
\tau(t) \geq t
$$

for $t \geq t_{0}$.

Theorem 2.1 Let (2.1) hold and $\lim _{t \rightarrow+\infty} \tau(t)=+\infty$. Suppose that there exist functions $H \in \mathcal{W}$ and $\rho_{1} \in C^{1}\left(\left[t_{0},+\infty\right), \mathbb{R}\right)$ such that, for some $\beta \geq 1$,

$$
\limsup _{t \rightarrow+\infty} \frac{1}{H\left(t, T_{1}\right)} \int_{T_{1}}^{t}\left[H(t, s) \psi_{*}(s)-\frac{\beta}{4} \nu_{*}(s) r(s) h^{2}(t, s)\right] \mathrm{d} s=+\infty
$$

for all sufficiently large $t_{1} \geq t_{0}$ and for $T_{1}>t_{1}$, where

$$
\begin{aligned}
\psi_{*}(t):= & v_{*}(t)\left[\mu q(t) \frac{\int_{t_{1}}^{\tau(t)} \frac{1}{r(s) \exp \left(\int_{t_{0}}^{s} \frac{p(v)}{r(v)} \mathrm{d} v\right)} \mathrm{d} s}{\int_{t_{1}}^{t} \frac{1}{r(s) \exp \left(\int_{t_{0}}^{s} \frac{p(v)}{r(v)} \mathrm{d} v\right)} \mathrm{d} s}\right. \\
& \left.+r(t) \rho_{1}^{2}(t)-p(t) \rho_{1}(t)-\left(r(t) \rho_{1}(t)\right)^{\prime}\right]
\end{aligned}
$$


and

$$
\nu_{*}(t):=\exp \left[-2 \int^{t}\left(\rho_{1}(s)-\frac{p(s)}{2 r(s)}\right) \mathrm{d} s\right] .
$$

Then (1.1) is oscillatory.

Proof Let $x$ be a nonoscillatory solution of (1.1). Without loss of generality, we may assume that there exists $T_{0} \geq t_{0}$ such that $x(t)>0$ and $x(\tau(t))>0$ for all $t \geq T_{0}$. By virtue of (1.1), we have

$$
\left(r(t) x^{\prime}(t)\right)^{\prime}+p(t) x^{\prime}(t) \leq-\mu q(t) x(\tau(t)) \leq 0 \quad \text { for } t \geq T_{0}
$$

which yields

$$
\left(r(t) x^{\prime}(t) \exp \left(\int_{t_{0}}^{t} \frac{p(s)}{r(s)} \mathrm{d} s\right)\right)^{\prime} \leq 0 .
$$

Hence we have

$$
r(t) \exp \left(\int_{t_{0}}^{t} \frac{p(s)}{r(s)} \mathrm{d} s\right) x^{\prime}(t)>0
$$

or

$$
r(t) \exp \left(\int_{t_{0}}^{t} \frac{p(s)}{r(s)} \mathrm{d} s\right) x^{\prime}(t)<0
$$

for $t \geq t_{1} \geq T_{0}$. Now define the generalized Riccati substitution

$$
u(t):=v_{*}(t) r(t)\left[\frac{x^{\prime}(t)}{x(t)}+\rho_{1}(t)\right]
$$

We consider each of two cases separately.

Case I. Assume (2.7) holds. Then we have

$$
\begin{aligned}
x(t) & =x\left(t_{1}\right)+\int_{t_{1}}^{t} \frac{r(s) \exp \left(\int_{t_{0}}^{s} \frac{p(v)}{r(v)} \mathrm{d} v\right) x^{\prime}(s)}{r(s) \exp \left(\int_{t_{0}}^{s} \frac{p(v)}{r(v)} \mathrm{d} v\right)} \mathrm{d} s \\
& \geq x^{\prime}(t) r(t) \exp \left(\int_{t_{0}}^{t} \frac{p(s)}{r(s)} \mathrm{d} s\right) \int_{t_{1}}^{t} \frac{1}{r(s) \exp \left(\int_{t_{0}}^{s} \frac{p(v)}{r(v)} \mathrm{d} v\right)} \mathrm{d} s,
\end{aligned}
$$

which implies that

$$
\left(\frac{x(t)}{\int_{t_{1}}^{t} \frac{1}{r(s) \exp \left(\int_{t_{0}}^{s} \frac{p(v)}{r(v)} \mathrm{d} v\right)} \mathrm{d} s}\right)^{\prime} \leq 0 .
$$


Differentiating (2.9) yields

$$
\begin{aligned}
u^{\prime}(t)= & \frac{v_{*}^{\prime}(t)}{v_{*}(t)} u(t)+v_{*}(t) \frac{\left(r(t) x^{\prime}(t)\right)^{\prime}}{x(t)} \\
& -v_{*}(t) r(t)\left[\frac{u(t)}{v_{*}(t) r(t)}-\rho_{1}(t)\right]^{2}+v_{*}(t)\left(r(t) \rho_{1}(t)\right)^{\prime} .
\end{aligned}
$$

It follows from (1.1), (2.5), (2.10), and (2.11) that

$$
u^{\prime}(t) \leq-\psi_{*}(t)-\frac{u^{2}(t)}{v_{*}(t) r(t)}
$$

where $\psi_{*}$ is defined as in (2.4). Multiplying both sides of (2.12), with $t$ replaced by $s$, by $H(t, s)$, integrating with respect to $s$ from $T_{1}$ to $t$, we find, for all $\beta \geq 1$ and for all $t \geq T_{1} \geq t_{1}$,

$$
\begin{aligned}
& \int_{T_{1}}^{t} H(t, s) \psi_{*}(s) \mathrm{d} s+\int_{T_{1}}^{t} h(t, s)(H(t, s))^{\frac{1}{2}} u(s) \mathrm{d} s+\frac{1}{\beta} \int_{T_{1}}^{t} H(t, s) \frac{u^{2}(s)}{v_{*}(s) r(s)} \mathrm{d} s \\
& \quad \leq H\left(t, T_{1}\right) u\left(T_{1}\right)-\frac{\beta-1}{\beta} \int_{T_{1}}^{t} H(t, s) \frac{u^{2}(s)}{v_{*}(s) r(s)} \mathrm{d} s .
\end{aligned}
$$

Define now

$$
C:=\frac{u(s)}{\sqrt{\beta}} \frac{(H(t, s))^{\frac{1}{2}}}{\left(v_{*}(s) r(s)\right)^{\frac{1}{2}}} \quad \text { and } \quad D:=-\frac{\sqrt{\beta}}{2} h(t, s)\left(v_{*}(s) r(s)\right)^{\frac{1}{2}} .
$$

Applying the inequality

$$
C^{2}-2 C D \geq-D^{2}
$$

we have

$$
h(t, s)(H(t, s))^{\frac{1}{2}} u(s)+\frac{1}{\beta} H(t, s) \frac{u^{2}(s)}{v_{*}(s) r(s)} \geq-\frac{\beta}{4} \nu_{*}(s) r(s) h^{2}(t, s) .
$$

Hence, by the latter inequality and (2.13), we obtain

$$
\begin{aligned}
& \int_{T_{1}}^{t}\left[H(t, s) \psi_{*}(s)-\frac{\beta}{4} v_{*}(s) r(s) h^{2}(t, s)\right] \mathrm{d} s \\
& \quad \leq H\left(t, T_{1}\right) u\left(T_{1}\right)-\frac{\beta-1}{\beta} \int_{T_{1}}^{t} H(t, s) \frac{u^{2}(s)}{v_{*}(s) r(s)} \mathrm{d} s,
\end{aligned}
$$

which contradicts (2.3).

Case II. Assume (2.8) holds. Recalling that $x^{\prime}<0$ and $\tau(t) \leq t$, we have $x(\tau(t)) \geq x(t)$. Using similar proof of the case where (2.7) holds and the fact that

$$
\frac{\int_{t_{1}}^{\tau(t)} \frac{1}{r(s) \exp \left(\int_{t_{0}}^{s} \frac{p(v)}{r(v)} \mathrm{d} v\right)} \mathrm{d} s}{\int_{t_{1}}^{t} \frac{1}{r(s) \exp \left(\int_{t_{0}}^{s} \frac{p(v)}{r(v)} \mathrm{d} v\right)} \mathrm{d} s} \leq 1,
$$

one has (2.15), which contradicts (2.3). This completes the proof. 
Theorem 2.2 Let (2.1) hold and $\lim _{t \rightarrow+\infty} \tau(t)=+\infty$. Suppose that there exist functions $H \in \mathcal{W}, \rho_{1} \in \mathrm{C}^{1}\left(\left[t_{0},+\infty\right), \mathbb{R}\right)$, and $\phi_{*} \in \mathrm{C}\left(\left[t_{0},+\infty\right), \mathbb{R}\right)$ such that, for all sufficiently large $T>t_{1}$ and for some $\beta>1$,

$$
0<\inf _{s \geq t_{0}}\left[\liminf _{t \rightarrow+\infty} \frac{H(t, s)}{H\left(t, t_{0}\right)}\right] \leq+\infty
$$

and

$$
\limsup _{t \rightarrow+\infty} \frac{1}{H(t, T)} \int_{T}^{t}\left[H(t, s) \psi_{*}(s)-\frac{\beta}{4} v_{*}(s) r(s) h^{2}(t, s)\right] \mathrm{d} s \geq \phi_{*}(T),
$$

where $\psi_{*}$ and $v_{*}$ are as in Theorem 2.1. If

$$
\int_{t_{0}}^{+\infty} \frac{\left(\phi_{*+}(s)\right)^{2}}{v_{*}(s) r(s)} \mathrm{d} s=+\infty
$$

where $\phi_{*+}(t):=\max \left\{\phi_{*}(t), 0\right\}$, then $(1.1)$ is oscillatory.

Proof Without loss of generality, assume again that (1.1) possesses a solution $x$ such that $x(t)>0$ and $x(\tau(t))>0$ on $\left[T_{0},+\infty\right)$ for some $T_{0} \geq t_{0}$. Proceeding as in the proof of Theorem 2.1, we arrive at inequality (2.15), which yields, for all $t>T_{1}$ and for any $\beta \geq 1$,

$$
\begin{aligned}
\phi_{*}\left(T_{1}\right) & \leq \limsup _{t \rightarrow+\infty} \frac{1}{H\left(t, T_{1}\right)} \int_{T_{1}}^{t}\left[H(t, s) \psi_{*}(s)-\frac{\beta}{4} v_{*}(s) r(s) h^{2}(t, s)\right] \mathrm{d} s \\
& \leq u\left(T_{1}\right)-\frac{\beta-1}{\beta} \liminf _{t \rightarrow+\infty} \frac{1}{H\left(t, T_{1}\right)} \int_{T_{1}}^{t} H(t, s) \frac{u^{2}(s)}{v_{*}(s) r(s)} \mathrm{d} s .
\end{aligned}
$$

The latter inequality implies that, for all $t>T_{1}$ and for all $\beta \geq 1$,

$$
\phi_{*}\left(T_{1}\right)+\frac{\beta-1}{\beta} \liminf _{t \rightarrow+\infty} \frac{1}{H\left(t, T_{1}\right)} \int_{T_{1}}^{t} H(t, s) \frac{u^{2}(s)}{v_{*}(s) r(s)} \mathrm{d} s \leq u\left(T_{1}\right) .
$$

Consequently,

$$
\phi_{*}\left(T_{1}\right) \leq u\left(T_{1}\right)
$$

and

$$
\liminf _{t \rightarrow+\infty} \frac{1}{H\left(t, T_{1}\right)} \int_{T_{1}}^{t} H(t, s) \frac{u^{2}(s)}{v_{*}(s) r(s)} \mathrm{d} s \leq \frac{\beta}{\beta-1}\left(u\left(T_{1}\right)-\phi_{*}\left(T_{1}\right)\right)<+\infty
$$

Assume now that

$$
\int_{T_{1}}^{+\infty} \frac{u^{2}(s)}{v_{*}(s) r(s)} \mathrm{d} s=+\infty
$$

Condition (2.16) implies the existence of $\vartheta>0$ such that

$$
\inf _{s \geq t_{0}}\left[\liminf _{t \rightarrow+\infty} \frac{H(t, s)}{H\left(t, t_{0}\right)}\right]>\vartheta
$$


It follows from (2.21) that, for any positive constant $\eta$, there exists $T_{2}>T_{1}$ such that, for all $t \geq T_{2}$,

$$
\int_{T_{1}}^{t} \frac{u^{2}(s)}{v_{*}(s) r(s)} \mathrm{d} s \geq \frac{\eta}{\vartheta} .
$$

Using integration by parts and (2.23), we have, for all $t \geq T_{2}$,

$$
\begin{aligned}
& \frac{1}{H\left(t, T_{1}\right)} \int_{T_{1}}^{t} H(t, s) \frac{u^{2}(s)}{v_{*}(s) r(s)} \mathrm{d} s \\
& =\frac{1}{H\left(t, T_{1}\right)} \int_{T_{1}}^{t} H(t, s) \mathrm{d}\left[\int_{T_{1}}^{s} \frac{u^{2}(\xi)}{v_{*}(\xi) r(\xi)} \mathrm{d} \xi\right] \\
& =\frac{1}{H\left(t, T_{1}\right)} \int_{T_{1}}^{t}\left[\int_{T_{1}}^{s} \frac{u^{2}(\xi)}{v_{*}(\xi) r(\xi)} \mathrm{d} \xi\right]\left[-\frac{\partial H(t, s)}{\partial s}\right] \mathrm{d} s \\
& \geq \frac{\eta}{\vartheta} \frac{1}{H\left(t, T_{1}\right)} \int_{T_{2}}^{t}\left[-\frac{\partial H(t, s)}{\partial s}\right] \mathrm{d} s=\frac{\eta}{\vartheta} \frac{H\left(t, T_{2}\right)}{H\left(t, T_{1}\right)} \geq \frac{\eta}{\vartheta} \frac{H\left(t, T_{2}\right)}{H\left(t, t_{0}\right)} .
\end{aligned}
$$

By virtue of (2.22), there exists $T_{3} \geq T_{2}$ such that, for all $t \geq T_{3}$,

$$
\frac{H\left(t, T_{2}\right)}{H\left(t, t_{0}\right)} \geq \vartheta,
$$

which yields

$$
\frac{1}{H\left(t, T_{1}\right)} \int_{T_{1}}^{t} H(t, s) \frac{u^{2}(s)}{v_{*}(s) r(s)} \mathrm{d} s \geq \eta, \quad t \geq T_{3} .
$$

Since $\eta$ is an arbitrary positive constant,

$$
\liminf _{t \rightarrow+\infty} \frac{1}{H\left(t, T_{1}\right)} \int_{T_{1}}^{t} H(t, s) \frac{u^{2}(s)}{v_{*}(s) r(s)} \mathrm{d} s=+\infty,
$$

and the latter contradicts (2.20). Consequently,

$$
\int_{T_{1}}^{+\infty} \frac{u^{2}(s)}{v_{*}(s) r(s)} \mathrm{d} s<+\infty
$$

and, by virtue of (2.19),

$$
\int_{T_{1}}^{+\infty} \frac{\left(\phi_{*+}(s)\right)^{2}}{v_{*}(s) r(s)} \mathrm{d} s \leq \int_{T_{1}}^{+\infty} \frac{u^{2}(s)}{v_{*}(s) r(s)} \mathrm{d} s<+\infty,
$$

which contradicts (2.18). This completes the proof.

Theorem 2.3 Let (2.2) hold and

$$
\int^{+\infty} \frac{1}{r(s)} \exp \left(-\int_{t_{0}}^{s} \frac{p(t)}{r(t)} \mathrm{d} t\right) \mathrm{d} s<+\infty .
$$


Suppose that there exist functions $H \in \mathcal{W}$ and $\rho_{2} \in C^{1}\left(\left[t_{0},+\infty\right), \mathbb{R}\right)$ such that, for some $\beta \geq 1$,

$$
\limsup _{t \rightarrow+\infty} \frac{1}{H\left(t, t_{0}\right)} \int_{t_{0}}^{t}\left[H(t, s) \varphi_{*}(s)-\frac{\beta}{4} v(s) r(s) h^{2}(t, s)\right] \mathrm{d} s=+\infty,
$$

where

$$
\varphi_{*}(t):=v(t)\left[\mu q(t) \frac{\int_{\tau(t)}^{+\infty} \frac{1}{r(s) \exp \left(\int_{t_{0}}^{s} \frac{p(z)}{r(z)} \mathrm{d} z\right)} \mathrm{d} s}{\int_{t}^{+\infty} \frac{1}{r(s) \exp \left(\int_{t_{0}}^{s} \frac{p(z)}{r(z)} \mathrm{d} z\right)} \mathrm{d} s}+r(t) \rho_{2}^{2}(t)-p(t) \rho_{2}(t)-\left(r(t) \rho_{2}(t)\right)^{\prime}\right]
$$

and

$$
v(t):=\exp \left[-2 \int^{t}\left(\rho_{2}(s)-\frac{p(s)}{2 r(s)}\right) \mathrm{d} s\right]
$$

Then (1.1) is oscillatory.

Proof Let $x$ be a nonoscillatory solution of (1.1). Without loss of generality, we may assume that there exists $T_{0} \geq t_{0}$ such that $x(t)>0$ for all $t \geq T_{0}$. From the proof of Theorem 2.1, we have (2.6) and either (2.7) or (2.8) for $t \geq t_{1} \geq T_{0}$. We define the generalized Riccati substitution

$$
u(t):=v(t) r(t)\left[\frac{x^{\prime}(t)}{x(t)}+\rho_{2}(t)\right] .
$$

Case I. Assume (2.7) holds. Differentiating (2.28), we have

$$
u^{\prime}(t)=\frac{v^{\prime}(t)}{v(t)} u(t)+v(t) \frac{\left(r(t) x^{\prime}(t)\right)^{\prime}}{x(t)}-v(t) r(t)\left[\frac{u(t)}{v(t) r(t)}-\rho_{2}(t)\right]^{2}+v(t)\left(r(t) \rho_{2}(t)\right)^{\prime}
$$

It follows from (1.1), (2.27), and (2.29) that

$$
u^{\prime}(t) \leq-\varphi(t)-\frac{u^{2}(t)}{v(t) r(t)}
$$

where

$$
\varphi(t):=v(t)\left[\mu q(t)+r(t) \rho_{2}^{2}(t)-p(t) \rho_{2}(t)-\left(r(t) \rho_{2}(t)\right)^{\prime}\right] .
$$

Multiplying both sides of (2.30), with $t$ replaced by $s$, by $H(t, s)$, integrating with respect to $s$ from $T_{1}$ to $t$, we find, for all $\beta \geq 1$ and for all $t \geq T_{1} \geq t_{1}$,

$$
\begin{aligned}
& \int_{T_{1}}^{t} H(t, s) \varphi(s) \mathrm{d} s+\int_{T_{1}}^{t} h(t, s)(H(t, s))^{\frac{1}{2}} u(s) \mathrm{d} s+\frac{1}{\beta} \int_{T_{1}}^{t} H(t, s) \frac{u^{2}(s)}{v(s) r(s)} \mathrm{d} s \\
& \quad \leq H\left(t, T_{1}\right) u\left(T_{1}\right)-\frac{\beta-1}{\beta} \int_{T_{1}}^{t} H(t, s) \frac{u^{2}(s)}{v(s) r(s)} \mathrm{d} s .
\end{aligned}
$$


Now define

$$
C_{*}:=\frac{u(s)}{\sqrt{\beta}} \frac{(H(t, s))^{\frac{1}{2}}}{(v(s) r(s))^{\frac{1}{2}}} \quad \text { and } \quad D_{*}:=-\frac{\sqrt{\beta}}{2} h(t, s)(v(s) r(s))^{\frac{1}{2}} .
$$

Applying inequality (2.14) (replace $C$ and $D$ with $C_{*}$ and $D_{*}$ ), we have

$$
h(t, s)(H(t, s))^{\frac{1}{2}} u(s)+\frac{1}{\beta} H(t, s) \frac{u^{2}(s)}{v(s) r(s)} \geq-\frac{\beta}{4} v(s) r(s) h^{2}(t, s) .
$$

Hence, by the latter inequality and (2.31), we have

$$
\begin{aligned}
& \int_{T_{1}}^{t}\left[H(t, s) \varphi(s)-\frac{\beta}{4} v(s) r(s) h^{2}(t, s)\right] \mathrm{d} s \\
& \quad \leq H\left(t, T_{1}\right) u\left(T_{1}\right)-\frac{\beta-1}{\beta} \int_{T_{1}}^{t} H(t, s) \frac{u^{2}(s)}{v(s) r(s)} \mathrm{d} s .
\end{aligned}
$$

Using monotonicity of $H$, we conclude that, for all $t \geq T_{1}$,

$$
\int_{T_{1}}^{t}\left[H(t, s) \varphi(s)-\frac{\beta}{4} v(s) r(s) h^{2}(t, s)\right] \mathrm{d} s \leq H\left(t, T_{1}\right)\left|u\left(T_{1}\right)\right| \leq H\left(t, t_{0}\right)\left|u\left(T_{1}\right)\right| .
$$

Thus

$$
\int_{t_{0}}^{t}\left[H(t, s) \varphi(s)-\frac{\beta}{4} v(s) r(s) h^{2}(t, s)\right] \mathrm{d} s \leq H\left(t, t_{0}\right)\left[\left|u\left(T_{1}\right)\right|+\int_{t_{0}}^{T_{1}}|\varphi(s)| \mathrm{d} s\right] .
$$

Hence we have

$$
\limsup _{t \rightarrow+\infty} \frac{1}{H\left(t, t_{0}\right)} \int_{t_{0}}^{t}\left[H(t, s) \varphi(s)-\frac{\beta}{4} v(s) r(s) h^{2}(t, s)\right] \mathrm{d} s \leq\left|u\left(T_{1}\right)\right|+\int_{t_{0}}^{T_{1}}|\varphi(s)| \mathrm{d} s<+\infty
$$

which contradicts (2.25) due to the fact that $\varphi_{*}(t) \leq \varphi(t)$, where $\varphi_{*}$ is defined as in (2.26).

Case II. Assume (2.8) holds. From (2.6), we have

$$
x^{\prime}(s) \leq \frac{r(t) \exp \left(\int_{t_{0}}^{t} \frac{p(z)}{r(z)} \mathrm{d} z\right)}{r(s) \exp \left(\int_{t_{0}}^{s} \frac{p(z)}{r(z)} \mathrm{d} z\right)} x^{\prime}(t), \quad s \geq t .
$$

Hence we get

$$
x(l)-x(t) \leq x^{\prime}(t) r(t) \exp \left(\int_{t_{0}}^{t} \frac{p(z)}{r(z)} \mathrm{d} z\right) \int_{t}^{l} \frac{1}{r(s) \exp \left(\int_{t_{0}}^{s} \frac{p(z)}{r(z)} \mathrm{d} z\right)} \mathrm{d} s .
$$

Letting $l \rightarrow+\infty$, we obtain

$$
x(t) \geq-x^{\prime}(t) r(t) \exp \left(\int_{t_{0}}^{t} \frac{p(z)}{r(z)} \mathrm{d} z\right) \int_{t}^{+\infty} \frac{1}{r(s) \exp \left(\int_{t_{0}}^{s} \frac{p(z)}{r(z)} \mathrm{d} z\right)} \mathrm{d} s .
$$


This inequality yields

$$
\left(\frac{x(t)}{\int_{t}^{+\infty} \frac{1}{r(s) \exp \left(\int_{t_{0}}^{s} \frac{p(z)}{r(z)} \mathrm{d} z\right)} \mathrm{d} s}\right)^{\prime} \geq 0,
$$

and so

$$
\frac{x(\tau(t))}{x(t)} \geq \frac{\int_{\tau(t)}^{+\infty} \frac{1}{r(s) \exp \left(\int_{t_{0}}^{s} \frac{p(z)}{r(z)} \mathrm{d} z\right)} \mathrm{d} s}{\int_{t}^{+\infty} \frac{1}{r(s) \exp \left(\int_{t_{0}}^{s} \frac{p(z)}{r(z)} \mathrm{d} z\right)} \mathrm{d} s} .
$$

The rest of the proof is similar to that of the case where (2.7) holds. Then one can get a contradiction to (2.25). This completes the proof.

On the basis of Theorem 2.3, similar as in the proof of Theorem 2.2, we have the following result immediately.

Theorem 2.4 Let (2.2) and (2.24) hold. Suppose that there exist functions $H \in \mathcal{W}, \rho_{2} \in$ $\mathrm{C}^{1}\left(\left[t_{0},+\infty\right), \mathbb{R}\right)$, and $\phi \in \mathrm{C}\left(\left[t_{0},+\infty\right), \mathbb{R}\right)$ such that, for all $T \geq t_{0}$ and for some $\beta>1$, one has (2.16) and

$$
\limsup _{t \rightarrow+\infty} \frac{1}{H(t, T)} \int_{T}^{t}\left[H(t, s) \varphi_{*}(s)-\frac{\beta}{4} v(s) r(s) h^{2}(t, s)\right] \mathrm{d} s \geq \phi(T),
$$

where $\varphi_{*}$ and $v$ are as in Theorem 2.3. If

$$
\int_{t_{0}}^{+\infty} \frac{\left(\phi_{+}(s)\right)^{2}}{v(s) r(s)} \mathrm{d} s=+\infty
$$

where $\phi_{+}(t):=\max \{\phi(t), 0\}$, then (1.1) is oscillatory.

Remark 2.1 Efficient oscillation tests can be derived from Theorems 2.1-2.4 with different choices of the functions $H, \rho_{1}$, and $\rho_{2}$. For example, for $(t, s) \in \mathbb{D}$, Kamenev's weight function $H$ defined by $H(t, s)=(t-s)^{m}$, where $m \geq 1$, belongs to the class $\mathcal{W}$. The details are left to the reader.

\section{Applications and discussion}

The following three examples illustrate applications of theoretical results in the previous section.

Example 3.1 For $t \geq 1$, consider a second-order ordinary damped differential equation

$$
x^{\prime \prime}(t)+\frac{1}{t} x^{\prime}(t)+\frac{1}{t^{2}} x(t)=0,
$$

where $r(t)=1, p(t)=1 / t, q(t)=1 / t^{2}, f(x)=x$, and $\tau(t)=t$. Letting $\mu=1, \rho_{1}(t)=0$, and $H(t, s)=(t-s)^{2}$, then $v_{*}(t)=t, h^{2}(t, s)=4$, and so $\psi_{*}(t)=1 / t$ and

$$
\begin{gathered}
\limsup _{t \rightarrow+\infty} \frac{1}{H\left(t, T_{1}\right)} \int_{T_{1}}^{t}\left[H(t, s) \psi_{*}(s)-\frac{\beta}{4} \nu_{*}(s) r(s) h^{2}(t, s)\right] \mathrm{d} s \\
\quad=\limsup _{t \rightarrow+\infty} \frac{1}{t^{2}} \int_{T_{1}}^{t}\left[\frac{(t-s)^{2}}{s}-\beta s\right] \mathrm{d} s=+\infty .
\end{gathered}
$$


Hence, by Theorem 2.1, equation (3.1) is oscillatory. As a matter of fact, one such solution is $x(t)=\sin (\ln t)$.

Example 3.2 For $t \geq 1$, consider a second-order delay damped differential equation

$$
x^{\prime \prime}(t)-x^{\prime}(t)+\sqrt{2} x\left(t-\frac{7 \pi}{4}\right)=0,
$$

where $r(t)=1, p(t)=-1, q(t)=\sqrt{2}, f(x)=x$, and $\tau(t)=t-7 \pi / 4$. Letting $\mu=1, \rho_{1}(t)=-1 / 2$, and $H(t, s)=(t-s)^{2}$, then $v_{*}(t)=1, h^{2}(t, s)=4$, and so $\psi_{*}(t)>3 / 4$ and

$$
\begin{aligned}
& \limsup _{t \rightarrow+\infty} \frac{1}{H\left(t, T_{1}\right)} \int_{T_{1}}^{t}\left[H(t, s) \psi_{*}(s)-\frac{\beta}{4} v_{*}(s) r(s) h^{2}(t, s)\right] \mathrm{d} s \\
& \quad \geq \limsup _{t \rightarrow+\infty} \frac{1}{t^{2}} \int_{T_{1}}^{t}\left[\frac{3(t-s)^{2}}{4}-\beta\right] \mathrm{d} s=+\infty .
\end{aligned}
$$

Hence, by Theorem 2.1, equation (3.2) is oscillatory. As a matter of fact, one such solution is $x(t)=\sin t$.

Example 3.3 For $t \geq 1$, consider a second-order advanced damped differential equation

$$
x^{\prime \prime}(t)+x^{\prime}(t)+x(t+1)=0,
$$

where $r(t)=1, p(t)=1, q(t)=1, f(x)=x$, and $\tau(t)=t+1$. Letting $\mu=1, \rho_{2}(t)=1 / 2$, and $H(t, s)=(t-s)^{2}$, then $v(t)=1, h^{2}(t, s)=4$, and so $\varphi_{*}(t)=\mathrm{e}^{-1}-1 / 4$ and

$$
\begin{gathered}
\limsup _{t \rightarrow+\infty} \frac{1}{H\left(t, T_{1}\right)} \int_{T_{1}}^{t}\left[H(t, s) \varphi_{*}(s)-\frac{\beta}{4} v(s) r(s) h^{2}(t, s)\right] \mathrm{d} s \\
=\limsup _{t \rightarrow+\infty} \frac{1}{t^{2}} \int_{T_{1}}^{t}\left[\left(\mathrm{e}^{-1}-\frac{1}{4}\right)(t-s)^{2}-\beta\right] \mathrm{d} s=+\infty .
\end{gathered}
$$

Hence, by Theorem 2.3, equation (3.3) is oscillatory.

Remark 3.1 In this paper, we present some new oscillation criteria for the differential equation with a linear damping term (1.1). Our theorems can be applied to the cases where $p \geq 0, p \leq 0$, or $p$ is an oscillatory function. Furthermore, the main results can be applied to the cases where the deviating argument $\tau$ is delayed or advanced. On the other hand, we do not need to require the assumption that $\tau^{\prime}(t)>0$ for $t \geq t_{0}$. Hence, the results obtained supplement and improve those reported in $[8,15]$.

Remark 3.2 Note that when $\tau(t) \equiv t$, Theorems 2.1 and 2.2 include [20, Theorem 17] and [20, Theorem 19], respectively. On the basis of assumption (2.24), Theorems 2.3 and 2.4 include [20, Theorem 17] and [20, Theorem 19], respectively. 
Authors' contributions

All authors read and approved the final manuscript.

\section{Author details}

${ }^{1}$ School of Control Science and Engineering, Shandong University, Jinan, Shandong 250061, P.R. China. ${ }^{2}$ Department of Physics, Changji University, Changji, Xinjiang 831100, P.R. China.

\section{Acknowledgements}

The authors would like to thank the editors and referees for their thoughtful review of this manuscript and their insightful comments used to improve the quality of this paper. This research is supported by the National Key Basic Research Program of P.R. China (2013CB035604), NNSF of P.R. China (Grant Nos. 61034007, 51277116, 51107069, 61304029), and NSF of Xinjiang (Grant No. 201318101-16)

Received: 8 May 2013 Accepted: 30 September 2013 Published: 19 Nov 2013

\section{References}

1. Agarwal, RP, Bohner, M, Li, WT: Nonoscillation and Oscillation: Theory for Functional Differential Equations. Monographs and Textbooks in Pure and Applied Mathematics, vol. 267. Dekker, New York (2004)

2. Agarwal, RP, Grace, SR, O'Regan, D: Oscillation Theory for Difference and Functional Differential Equations. Kluwer Academic, Dordrecht (2000)

3. Aktaş, MF, Çakmak, D, Tiryaki, A: On the qualitative behaviors of solutions of third-order nonlinear functional differential equations. Appl. Math. Lett. 24, 1849-1855 (2011)

4. Aktaş, MF, Tiryaki, A, Zafer, A: Oscillation criteria for third-order nonlinear functional differential equations. Appl. Math. Lett. 23, 756-762 (2010)

5. Baculíková, B, Džurina, J, Rogovchenko, YV: Oscillation of third order trinomial delay differential equations. Appl. Math. Comput. 218, 7023-7033 (2012)

6. Candan, T: Oscillation criteria for second-order nonlinear neutral dynamic equations with distributed deviating arguments on time scales. Adv. Differ. Equ. 2013, 1-8 (2013)

7. Candan, T: Oscillation of second-order nonlinear neutral dynamic equations on time scales with distributed deviating arguments. Comput. Math. Appl. 62, 4118-4125 (2011)

8. Bohner, M, Saker, SH: Oscillation of damped second order nonlinear delay differential equations of Emden-Fowler type. Adv. Dyn. Syst. Appl. 1, 163-182 (2006)

9. Džurina, J, Komariková, R: Asymptotic properties of third-order delay trinomial differential equations. Abstr. Appl. Anal. 2011, Article ID 730128 (2011)

10. Grace, SR, Lalli, BS, Yeh, CC: Oscillation theorems for nonlinear second order differential equations with a nonlinear damping term. SIAM J. Math. Anal. 15, 1082-1093 (1984)

11. Kirane, $M$, Rogovchenko, YV: Oscillation results for a second order damped differential equation with nonmonotonous nonlinearity. J. Math. Anal. Appl. 250, 118-138 (2000)

12. Kirane, M, Rogovchenko, YV: On oscillation of nonlinear second order differential equation with damping term. Appl. Math. Comput. 117, 177-192 (2001)

13. Li, T, Rogovchenko, YV, Tang, S: Oscillation of second-order nonlinear differential equations with damping. Math. Slovaca (2012, in press)

14. Li, WT, Agarwal, RP: Interval oscillation criteria for second order nonlinear differential equations with damping. Comput. Math. Appl. 40, 217-230 (2000)

15. Liu, S, Zhang, Q, Yu, Y: Oscillation of even-order half-linear functional differential equations with damping. Comput. Math. Appl. 61, 2191-2196 (2011)

16. Rogovchenko, SP, Rogovchenko, YV: Oscillation of differential equations with damping. Dyn. Contin. Discrete Impuls. Syst., Ser. A Math. Anal. 10, 447-461 (2003)

17. Rogovchenko, SP, Rogovchenko, YV: Oscillation theorems for differential equations with a nonlinear damping term. J. Math. Anal. Appl. 279, 121-134 (2003)

18. Rogovchenko, YV: Oscillation criteria for second order nonlinear perturbed differential equations. J. Math. Anal. Appl. 215, 334-357 (1997)

19. Rogovchenko, YV: Oscillation theorems for second-order equations with damping. Nonlinear Anal. 41, 1005-1028 (2000)

20. Rogovchenko, YV, Tuncay, F: Oscillation criteria for second-order nonlinear differential equations with damping. Nonlinear Anal. 69, 208-221 (2008)

21. Rogovchenko, YV, Tuncay, F: Oscillation theorems for a class of second order nonlinear differential equations with damping. Taiwan. J. Math. 13, 1909-1928 (2009)

22. Şenel, MT, Temtek, P: On behaviour of solutions for third order nonlinear ordinary differential equations with damping terms. J. Comput. Anal. Appl. 11, 346-355 (2009)

23. Tiryaki, A, Aktaş, MF: Oscillation criteria of a certain class of third order nonlinear delay differential equations with damping. J. Math. Anal. Appl. 325, 54-68 (2007)

24. Tiryaki, A, Zafer, A: Interval oscillation of a general class of second-order nonlinear differential equations with nonlinear damping. Nonlinear Anal. 60, 49-63 (2005)

25. Yan, J: Oscillation theorems for second order linear differential equations with damping. Proc. Am. Math. Soc. 98, 276-282 (1986)

26. Yeh, CC: Oscillation theorems for nonlinear second order differential equations with damping term. Proc. Am. Math. Soc. 84, 397-402 (1982)

27. Kamenev, IV: An integral criterion for oscillation of linear differential equations. Mat. Zametki 23, 249-251 (1978)

10.1186/1687-1847-2013-326

Cite this article as: Fu et al.: Oscillation of second-order damped differential equations. Advances in Difference Equations 2013, 2013:326 